\title{
CENTRALISER DIMENSION OF FREE PARTIALLY COMMUTATIVE NILPOTENT GROUPS OF CLASS 2
}

\author{
VIKKI A. BLATHERWICK \\ School of Mathematics and Statistics Newcastle University, Newcastle-upon-tyne, NE1 7RU \\ e-mail:v.a.blatherwick@newcastle.ac.uk
}

(Received 25 April, 2007; accepted 7 October, 2007)

\begin{abstract}
In an effort to extend the theory of algebraic geometry over groups beyond free groups, Duncan, Kazatchkov and Remeslennikov have studied the notion of centraliser dimension for free partially commutative groups. In this paper we consider the centraliser dimension of free partially commutative nilpotent groups of class 2 , showing that a free partially commutative nilpotent group of class 2 with non-commutation graph $\Gamma$ has the same centraliser dimension as the free partially commutative group represented by the non-commutation graph $\Gamma$.
\end{abstract}

2000 Mathematics Subject Classification. Primary 20F18; 20 F36.

1. Introduction. There has been much interest in recent years in the development of algebraic geometry over groups (see $[\mathbf{1}, \mathbf{1 0}, \mathbf{1 2}]$ ), inspired by various successes in the study of equations over groups, such as Makanin's algorithm for deciding whether a system of equations over a free group has a solution, and the solution of Tarski's problem concerning the elementary theory of free groups by Kharlampovich and Myasnikov [9], and, independently, Sela [16]. In [3], Chiswell and Remeslennikov showed that if $V$ is an irreducible algebraic set defined by a one-variable system of equations over a non-abelian free group, $F$, then either $V=F$, or $V$ is a point, or there exist elements $f, g, h \in F$ such that $V=f C_{F}(g) h$, where $C_{F}(g)$ is the centraliser in $F$ of $g$. It is therefore believed that the investigation of the structure of the centraliser lattice of a group is a first step in the construction of algebraic geometry over that group. The centraliser dimension of a group is defined to be the height of its centraliser lattice, a concept which has been studied in numerous papers, for example [15], [11], [2], [13] and [4]. To extend the theory of algebraic geometry over groups beyond free groups, Duncan, Kazatchkov and Remeslennikov [5] have studied the notion of centraliser dimension for free partially commutative groups, also known as graph groups, trace groups or right-angle Artin groups.

Given an arbitrary variety of groups, $\mathcal{V}$, we may define the class of free partially commutative $\mathcal{V}$ groups in an obvious fashion. In this paper we consider the centraliser dimension of free partially commutative nilpotent groups of class 2 . We begin, in Section 3, by considering the form of powers and roots of group elements. We then investigate the structure of centralisers in free partially commutative nilpotent groups of class 2 in Section 4. In Section 5 we show that to calculate centraliser dimension in these groups we only need to consider chains of centralisers of generating elements. This result is then used to show that a free partially commutative nilpotent group of class 2 with non-commutation graph $\Gamma$ has the same centraliser dimension as the free partially commutative group represented by non-commutation graph $\Gamma$. 
2. Preliminaries. In this section, we introduce some preliminary definitions which we will use throughout the paper. For further information on nilpotent groups the reader is referred to the texts by Hall [7] and Neumann [14].

Let $G$ be a group. The commutator of two elements $g_{1}, g_{2} \in G$ is denoted by

$$
\left[g_{1}, g_{2}\right]=g_{1}^{-1} g_{2}^{-1} g_{1} g_{2} \text {. }
$$

For $H, K$ subgroups of $G$, we let $[H, K]$ denote the subgroup of $G$ generated by all commutators of the form $[h, k]$ for some $h \in H, k \in K$.

A group $G$ is nilpotent of class 2 if it has a central series of length 2, and no central series of $G$ has shorter length. In particular, the lower central series of $G$ is finite of length 2:

$$
G>[G, G]>[[G, G], G]=1 .
$$

Therefore a group $G$ is nilpotent of class 2 if and only if $[[G, G], G]=1$ and $[G, G] \neq 1$. In particular we may note that $[g, h]$ is in the centre of $G$ for all elements $g, h \in G$. This definition may be further refined to show that if $G$ has presentation $G=\langle X \mid R\rangle$, then $G$ is nilpotent of class 2 if and only if $G$ is non-abelian and $[[x, y], z]=1$ for all $x, y, z \in X$. Thus a free nilpotent group of class 2 has presentation

$$
G=\langle X \mid\{[[x, y], z]: x, y, z \in X\}\rangle .
$$

A group $G$ is said to be (free) partially commutative if it has a presentation $G=\langle X \mid R\rangle$ such that

$$
R \subseteq\{[x, y]: x, y \in X\}
$$

Further information on this class of groups may be found in [4], [5] and [6]. We say $G$ is a (free) partially commutative nilpotent group of class 2 if $G=\langle X \mid R\rangle$ where

$$
\begin{aligned}
& R=\{[[x, y], z]: x, y, z \in X\} \cup R^{\prime}, \\
& R^{\prime} \subsetneq\{[x, y]: x, y \in X\} .
\end{aligned}
$$

In both of these cases, we define the non-commutation graph, $\Gamma(G)$, of $G$ to be the graph with vertex set $X$, and with an edge connecting vertices $x_{i}$ and $x_{j}$ if and only if $\left[x_{i}, x_{j}\right] \notin R$.

3. Powers and Roots of Group Elements. Every element $g$ of the free nilpotent group of class 2 and rank $n$,

$$
\left\langle x_{1}, x_{2}, \ldots, x_{n} \mid\left\{[[x, y], z]: x, y, z \in\left\{x_{1}, x_{2}, \ldots, x_{n}\right\}\right\}\right\rangle
$$

may be written uniquely in the normal form

$$
\begin{aligned}
g & =x_{1}^{r_{1}} \ldots x_{n}^{r_{n}}\left[x_{1}, x_{2}\right]^{s_{1,2}} \ldots\left[x_{1}, x_{n}\right]^{s_{1, n}}\left[x_{2}, x_{3}\right]^{s_{2,3}} \ldots\left[x_{2}, x_{n}\right]^{s_{2, n}} \ldots\left[x_{n-1}, x_{n}\right]^{s_{n-1, n}}, \\
& =x_{1}^{r_{1}} x_{2}^{r_{2}} \ldots x_{n}^{r_{n}} \prod_{1 \leqslant i<j \leqslant n}\left[x_{i}, x_{j}\right]^{s_{i, j}}
\end{aligned}
$$

for some $r_{i}, s_{i, j} \in \mathbb{Z}$ (see Hall [8] for further details). 
Let

$$
\begin{aligned}
G & =\langle X \mid R\rangle, \\
& =\left\langle x_{1}, x_{2}, \ldots, x_{n} \mid\left\{\left[\left[x_{i}, x_{j}\right], x_{k}\right]: i, j, k \in\{1,2, \ldots, n\}\right\},\left[x_{i_{1}}, x_{j_{1}}\right], \ldots,\left[x_{i_{m}}, x_{j_{m}}\right]\right\rangle,
\end{aligned}
$$

be a partially commutative nilpotent group of class 2 .

LEMMA. Every element $g \in G$ may be written uniquely in the normal form

$$
g=x_{1}^{r_{1}} x_{2}^{r_{2}} \ldots x_{n}^{r_{n}} \prod_{\substack{1 \leqslant i<j \leqslant n \\\left[x_{i}, x_{j}\right] \neq 1}}\left[x_{i}, x_{j}\right]^{s_{i, j}}
$$

for some $r_{i}, s_{i, j} \in \mathbb{Z}$.

If

$$
h=x_{1}^{r_{1}^{\prime}} x_{2}^{r_{2}^{\prime}} \ldots x_{n}^{r_{n}^{\prime}} \prod_{\substack{1 \leqslant i<j \leqslant n \\\left[x_{i}, x_{j}\right] \neq 1}}\left[x_{i}, x_{j}\right]^{s_{i, j}^{\prime}}
$$

then gh has normal form

$$
g h=x_{1}^{r_{1}+r_{1}^{\prime}} x_{2}^{r_{2}+r_{2}^{\prime}} \ldots x_{n}^{r_{n}+r_{n}^{\prime}} \prod_{\substack{1 \leqslant i<j \leqslant n \\\left[x_{i}, x_{j}\right] \neq 1}}\left[x_{i}, x_{j}\right]^{s_{i, j}+s_{i, j}^{\prime}-r_{i}^{\prime} r_{j}} .
$$

For $k \in \mathbb{Z}$, the $k$ th power of $g$ has normal form

$$
g^{k}=x_{1}^{k r_{1}} x_{2}^{k r_{2}} \ldots x_{n}^{k r_{n}} \prod_{\substack{1 \leqslant i<j \leqslant n \\\left[x_{i}, x_{j}\right] \neq 1}}\left[x_{i}, x_{j}\right]^{k s_{i, j}-\frac{1}{2}(k-1) k r_{i} r_{j}}
$$

Proof. Let $G^{\prime}=\left\langle X \mid R_{G^{\prime}}\right\rangle$ be the free nilpotent group of class 2 generated by the set $X$, and let

$$
R^{\prime}=\left\{\left[x_{i_{1}}, x_{j_{1}}\right],\left[x_{i_{2}}, x_{j_{2}}\right], \ldots,\left[x_{i_{m}}, x_{j_{m}}\right]\right\},
$$

so that $G=\left\langle X \mid R_{G^{\prime}}, R^{\prime}\right\rangle$. Note that $R^{\prime}$ is a subset of the centre of $G^{\prime}$, and therefore the subgroup, $N$, of $G^{\prime}$ generated by $R^{\prime}$ is a normal subgroup of $G^{\prime}$. By Von Dyck's Theorem, there exists an epimorphism, $\phi: G^{\prime} \rightarrow G$ such that $\phi$ fixes every $x \in X$, and $\operatorname{ker}(\phi)=N$, the normal closure of $R^{\prime}$.

Since $G$ is a nilpotent group of class 2 , every element of $G$ may be written in the form (1). In addition, for each commutator $\left[x_{i}, x_{j}\right] \in R^{\prime}$ we may set $s_{i, j}=0$, since $\left[x_{i}, x_{j}\right]=1$. Therefore every element $g$ in $G$ may be written in the form

$$
g=x_{1}^{r_{1}} x_{2}^{r_{2}} \ldots x_{n}^{r_{n}} \prod_{\substack{1 \leqslant i<j \leqslant n \\\left[x_{i}, x_{j}\right] \neq 1}}\left[x_{i}, x_{j}\right]^{s_{i, j}}
$$

for some $r_{i}, s_{i, j} \in \mathbb{Z}$. It remains to show that this is a unique form for elements of $G$.

Let

$$
g=x_{1}^{r_{1}} \ldots x_{n}^{r_{n}} \prod_{\substack{1 \leqslant i<j \leqslant n \\\left[x_{i}, x_{j}\right] \neq 1}}\left[x_{i}, x_{j}\right]^{s_{i, j}}, \quad h=x_{1}^{r_{1}^{\prime}} \ldots x_{n}^{r_{n}^{\prime}} \prod_{\substack{1 \leqslant i<j \leqslant n \\\left[x_{i}, x_{j}\right] \neq 1}}\left[x_{i}, x_{j}\right]^{s_{i, j}^{\prime}}
$$


be elements of $G$ written in the form (2). We wish to write the element $g h$ in the form (2). First we note that for any group $H$, and for any two elements $a$ and $b$ of $H$, we have the relation

$$
b a=a b b^{-1} a^{-1} b a=a b[b, a]=a b[a, b]^{-1} .
$$

Now suppose that $a$ and $b$ are elements of $G$. Since $G$ is a nilpotent group of class 2, we may use the above relation to see that

$$
\begin{aligned}
b^{s} a^{r} & =b^{s-1} a b a^{r-1}[a, b]^{-1}, \\
& =a b^{s} a^{r-1}[a, b]^{-s}, \\
& =a^{r} b^{s}[a, b]^{-r s},
\end{aligned}
$$

for all $r, s \in \mathbb{N}$. Further note that

$$
\left[a, b^{-1}\right]=b^{-1}\left[a, b^{-1}\right] b=b^{-1} a^{-1} b a b^{-1} b=\left(a^{-1} b^{-1} a b\right)^{-1}=[a, b]^{-1},
$$

and similarly that $\left[a^{-1}, b\right]=[a, b]^{-1}$ and $\left[a^{-1}, b^{-1}\right]=[a, b]$. Therefore, for all $r, s \in \mathbb{N}$ and all $\varepsilon, \delta \in\{-1,1\}$, the relation

$$
\begin{aligned}
b^{\delta s} a^{\varepsilon r} & =a^{\varepsilon r} b^{\delta s}\left[a^{\varepsilon}, b^{\delta}\right]^{-r s} \\
& =a^{\varepsilon r} b^{\delta s}[a, b]^{-(\varepsilon r)(\delta s)}
\end{aligned}
$$

holds in $G$.

We now use this relation to rearrange elements of our group $G$ into the form (2). For all $i \in\{1,2, \ldots, n-1\}$ and all $j \in\{i+1, i+2, \ldots, n\}$, we see that

$$
x_{j}^{r_{j}} x_{i}^{r_{i}^{\prime}}=x_{i}^{r_{i}^{\prime}} x_{j}^{r_{j}}\left[x_{i}, x_{j}\right]^{-r_{i}^{\prime} r_{j}} .
$$

Therefore

$$
\begin{aligned}
x_{1}^{r_{1}} x_{2}^{r_{2}} \ldots x_{n}^{r_{n}} x_{1}^{r_{1}^{\prime}} x_{2}^{r_{2}^{\prime}} \ldots x_{n}^{r_{n}^{\prime}} & x_{1}^{r_{1}} x_{2}^{r_{2}} \ldots x_{n-1}^{r_{n-1}} x_{1}^{r_{1}^{\prime}} x_{n}^{r_{n}} x_{2}^{r_{2}^{\prime}} \ldots x_{n}^{r_{n}^{\prime}}\left[x_{1}, x_{n}\right]^{-r_{1}^{\prime} r_{n}}, \\
& =x_{1}^{r_{1}+r_{1}^{\prime}} x_{2}^{r_{2}} x_{3}^{r_{3}} \ldots x_{n}^{r_{n}} x_{2}^{r_{2}^{\prime}} x_{3}^{r_{3}^{\prime}} \ldots x_{n}^{r_{n}^{\prime}} \prod_{1<j \leqslant n}\left[x_{1}, x_{j}\right]^{-r_{1}^{\prime} r_{j}}, \\
& =x_{1}^{r_{1}+r_{1}^{\prime}} x_{2}^{r_{2}+r_{2}^{\prime}} \ldots x_{n}^{r_{n}+r_{n}^{\prime}} \prod_{1 \leqslant i<j \leqslant n}\left[x_{i}, x_{j}\right]^{-r_{i}^{\prime} r_{j}},
\end{aligned}
$$

which implies that

$$
g h=x_{1}^{r_{1}+r_{1}^{\prime}} x_{2}^{r_{2}+r_{2}^{\prime}} \ldots x_{n}^{r_{n}+r_{n}^{\prime}} \prod_{\substack{1 \leqslant i<j \leqslant n \\\left[x_{i}, x_{j}\right] \neq 1}}\left[x_{i}, x_{j}\right]^{s_{i, j}+s_{i, j}^{\prime}-r_{i}^{\prime} r_{j}} .
$$

Note that this is in form (2).

Now let $k \in \mathbb{Z}$ and consider the element $g^{k}$. We wish to show that

$$
g^{k}=x_{1}^{k r_{1}} x_{2}^{k r_{2}} \ldots x_{n}^{k r_{n}} \prod_{\substack{1 \leqslant i<j \leqslant n \\\left[x_{i}, x_{j}\right] \neq 1}}\left[x_{i}, x_{j}\right]^{k s_{i, j}-\frac{1}{2}(k-1) k r_{i} r_{j}},
$$


for all integers $k$. We begin with the case where $k$ is a positive integer, and assume that $g^{k}$ may be written in the above form, which is clearly true for $k=1$. Noting that $g^{k+1}=g^{k} g$, we may apply our earlier result for products to see that,

$$
\begin{aligned}
g^{k+1} & =x_{1}^{k r_{1}+r_{1}} x_{2}^{k r_{2}+r_{2}} \ldots x_{n}^{k r_{n}+r_{n}} \prod_{\substack{1 \leqslant i<j \leqslant n \\
\left[x_{i}, x_{j}\right] \neq 1}}\left[x_{i}, x_{j}\right]^{s_{i, j}+k s_{i, j}-\frac{1}{2}(k-1) k r_{i} r_{j}-k r_{i} r_{j}}, \\
& =x_{1}^{(k+1) r_{1}} x_{2}^{(k+1) r_{2}} \ldots x_{n}^{(k+1) r_{n}} \prod_{\substack{1 \leqslant i<j \leqslant n \\
\left[x_{i}, x_{j}\right] \neq 1}}\left[x_{i}, x_{j}\right]^{(k+1) s_{i, j}-\frac{1}{2} k(k+1) r_{i} r_{j}} .
\end{aligned}
$$

Therefore, by induction, $g^{k}$ is of the required form for all positive integers $k$. The result also clearly holds for $k=0$. Now let $k=-1$. Using our earlier technique for rearranging group elements, we see that

$$
\begin{aligned}
g^{-1} & =x_{n}^{-r_{n}} x_{n-1}^{-r_{n-1}} \ldots x_{1}^{-r_{1}} \prod_{\substack{1 \leqslant i<j \leqslant n \\
\left[x_{i}, x_{j}\right] \neq 1}}\left[x_{i}, x_{j}\right]^{-s_{i, j}}, \\
& =x_{1}^{-r_{1}} x_{2}^{-r_{2}} \ldots x_{n}^{-r_{n}} \prod_{\substack{1 \leqslant i<j \leqslant n \\
\left[x_{i}, x_{j}\right] \neq 1}}\left[x_{i}, x_{j}\right]^{-s_{i, j}-r_{i} r_{j}},
\end{aligned}
$$

and therefore the result holds for $k=-1$. Finally note that for any positive integer $k$, we have

$$
\begin{aligned}
g^{-k} & =\left(g^{k}\right)^{-1}, \\
& =x_{1}^{-k r_{1}} x_{2}^{-k r_{2}} \ldots x_{n}^{-k r_{n}} \prod_{\substack{1 \leqslant i<j \leqslant n \\
\left[x_{i}, x_{j}\right] \neq 1}}\left[x_{i}, x_{j}\right]^{-\left(k s_{i, j}-\frac{1}{2}(k-1) k r_{i} r_{j}\right)-k^{2} r_{i} r_{j}}, \\
& =x_{1}^{-k r_{1}} x_{2}^{-k r_{2}} \ldots x_{n}^{-k r_{n}} \prod_{\substack{1 \leqslant i<j \leqslant n \\
\left[x_{i}, x_{j}\right] \neq 1}}\left[x_{i}, x_{j}\right]^{-k s_{i, j}-\frac{1}{2}(-k-1)(-k) r_{i} r_{j}},
\end{aligned}
$$

and therefore $g^{k}$ is of the required form for all integers $k$.

Now suppose that $g=h$ in $G$, so that

$$
\begin{aligned}
g h^{-1} & =x_{1}^{r_{1}-r_{1}^{\prime}} x_{2}^{r_{2}-r_{2}^{\prime}} \ldots x_{n}^{r_{n}-r_{n}^{\prime}} \prod_{\substack{1 \leqslant i<j \leqslant n \\
\left[x_{i}, x_{j}\right] \neq 1}}\left[x_{i}, x_{j}\right]^{s_{i, j}-s_{i, j}^{\prime}-r_{i}^{\prime} r_{j}^{\prime}+r_{i}^{\prime} r_{j}}, \\
& =1 .
\end{aligned}
$$

Recall that there exists an epimorphism $\phi: G^{\prime} \rightarrow G: x_{i} \mapsto x_{i}$ such that $\operatorname{ker}(\phi)=N$, where $N$ is the subgroup of $G$ generated by $R^{\prime}$. This implies that the inverse image of $g h^{-1}$ under $\phi$ is

$$
\phi^{-1}\left(g h^{-1}\right)=\left\{\prod_{\left[x_{i}, x_{j}\right] \in R^{\prime}}\left[x_{i}, x_{j}\right]^{t_{i, j}} \mid t_{i, j} \in \mathbb{Z}\right\} .
$$


Since $\phi\left(g h^{-1}\right)=g h^{-1}=1$, this implies that the relation

$$
x_{1}^{r_{1}-r_{1}^{\prime}} x_{2}^{r_{2}-r_{2}^{\prime}} \ldots x_{n}^{r_{n}-r_{n}^{\prime}} \prod_{\substack{1 \leqslant i<j \leqslant n \\\left[x_{i}, x_{j} \notin \notin R^{\prime}\right.}}\left[x_{i}, x_{j}\right]^{s_{i j}-s_{i, j}^{\prime}-r_{i}^{\prime} r_{j}^{\prime}+r_{i}^{\prime} r_{j}}=\prod_{\substack{1 \leqslant i<j \leqslant n \\\left[x_{i}, x_{j}\right] \in R^{\prime}}}\left[x_{i}, x_{j}\right]^{t_{i, j}},
$$

holds in the group $G^{\prime}$ for some $t_{i, j} \in \mathbb{Z}$. By the uniqueness of the normal form for $G^{\prime}$, this implies

(i) $r_{i}-r_{i}^{\prime}=0$ for all $i \in\{1,2, \ldots, n\}$; and

(ii) $s_{i, j}-s_{i, j}^{\prime}-r_{i}^{\prime} r_{j}^{\prime}+r_{i}^{\prime} r_{j}=0$ for all $1 \leqslant i<j \leqslant n$ such that $\left[x_{i}, x_{j}\right] \notin R^{\prime}$.

Therefore $r_{i}=r_{i}^{\prime}$ for all $i \in\{1,2, \ldots, n\}$ and $s_{i, j}=s_{i, j}^{\prime}$ for all $1 \leqslant i<j \leqslant n$ such that $\left[x_{i}, x_{j}\right] \notin R^{\prime}$. This implies that (2) is a unique normal form for elements of a partially commutative nilpotent group of class 2.

Let $g, h$ be elements of $G$. If $g$ and $h$ are both (positive) powers of some common element, then clearly there exist positive integers $k_{1}, k_{2}$ such that $g^{k_{1}}=h^{k_{2}}$. We now show that the converse is also true.

LEMmA. Let $g, h$ be elements of $G$. For any $k_{1}, k_{2} \in \mathbb{N}$, if $g^{k_{1}}=h^{k_{2}}$ then $g$ and $h$ are both powers of a common element. Further, if, in addition, $k_{1}=k_{2}$ then $g=h$.

Proof. Let

$$
g=x_{1}^{r_{1}} \ldots x_{n}^{r_{n}} \prod_{\substack{1 \leqslant i<j \leqslant n \\\left[x_{i}, x_{j} \neq 1\right.}}\left[x_{i}, x_{j}\right]^{s_{i j}}, \quad h=x_{1}^{r_{1}^{\prime}} \ldots x_{n}^{r_{n}^{\prime}} \prod_{\substack{1 \leqslant i<j \leqslant n \\\left[x_{i}, x_{j}\right] \neq 1}}\left[x_{i}, x_{j}\right]^{s_{i j}},
$$

be normal forms for elements $g, h \in G$. Suppose that $g^{k_{1}}=h^{k_{2}}$ for some $k_{1}, k_{2} \in \mathbb{N}$. This implies that

(i) $k_{1} r_{i}=k_{2} r_{i}^{\prime}$ for all $i \in\{1,2, \ldots, n\}$; and,

(ii) for all $1 \leqslant i<j \leqslant n$ such that $\left[x_{i}, x_{j}\right] \neq 1$,

$$
k_{1} s_{i, j}-\frac{1}{2}\left(k_{1}-1\right) k_{1} r_{i} r_{j}=k_{2} s_{i, j}^{\prime}-\frac{1}{2}\left(k_{2}-1\right) k_{2} r_{i}^{\prime} r_{j}^{\prime}
$$

We may assume without loss of generality that $k_{1} \leqslant k_{2}$. Note that if $k_{1}=k_{2}$ then $r_{i}=r_{i}^{\prime}$ for all $i \in\{1,2, \ldots, n\}$, and further $s_{i, j}=s_{i, j}^{\prime}$ for $1 \leqslant i<j \leqslant n$ such that $\left[x_{i}, x_{j}\right] \neq 1$. Therefore, if $k_{1}=k_{2}$ then $g=h$. Further note that if $k_{1}$ and $k_{2}$ are not coprime, say $\left(k_{1}, k_{2}\right)=t>1$, then $k_{1}=\alpha_{1} t$ and $k_{2}=\alpha_{2} t$ for some $\alpha_{1}, \alpha_{2} \in \mathbb{N}$ with $\left(\alpha_{1}, \alpha_{2}\right)=1$. This implies that $\left(g^{\alpha_{1}}\right)^{t}=\left(h^{\alpha_{2}}\right)^{t}$, so that $g^{\alpha_{1}}=h^{\alpha_{2}}$. It remains to consider the possibility that $\left(k_{1}, k_{2}\right)=1$. In this case, $k_{1}$ divides $r_{i}^{\prime}$ and $k_{2}$ divides $r_{i}$ for all $i \in\{1,2, \ldots, n\}$.

Suppose $k_{2}$ is odd. For some $t_{i} \in \mathbb{Z}$ we have

$$
g=x_{1}^{k_{2} t_{1}} x_{2}^{k_{2} t_{2}} \ldots x_{n}^{k_{2} t_{n}} \prod_{\substack{1 \leqslant i<j \leqslant n \\\left[x_{i}, x_{j} \neq \neq 1\right.}}\left[x_{i}, x_{j}\right]^{s_{i j}}
$$

Now for

$$
g^{\prime}=x_{1}^{t_{1}} x_{2}^{t_{2}} \ldots x_{n}^{t_{n}} \prod_{\substack{1 \leq i<j \leqslant n \\\left[x_{i}, x_{j}\right] \neq 1}}\left[x_{i}, x_{j}\right]^{u_{i, j}}
$$


we have

$$
\left(g^{\prime}\right)^{k_{2}}=x_{1}^{k_{2} t_{1}} x_{2}^{k_{2} t_{2}} \ldots x_{n}^{k_{2} t_{n}} \prod_{\substack{1 \leqslant i<j \leqslant n \\\left[x_{i}, x_{j}\right] \neq 1}}\left[x_{i}, x_{j}\right]^{k_{2} u_{i, j}-\frac{1}{2}\left(k_{2}-1\right) k_{2} t_{i} t_{j}} .
$$

Therefore, $g=\left(g^{\prime}\right)^{k_{2}}$ for some element $g^{\prime}$ of $G$ if and only if there exist integers $u_{i, j}$ such that

$$
s_{i, j}=k_{2} u_{i, j}-\frac{1}{2}\left(k_{2}-1\right) k_{2} t_{i} t_{j}
$$

for all $1 \leqslant i<j \leqslant n$ such that $\left[x_{i}, x_{j}\right] \neq 1$.

We may rearrange equation (3) to give

$$
k_{1} s_{i, j}=k_{2} s_{i, j}^{\prime}-\frac{1}{2}\left(k_{2}-1\right) k_{2} r_{i}^{\prime} r_{j}^{\prime}+\frac{1}{2}\left(k_{1}-1\right) k_{1} r_{i} r_{j}
$$

for all $1 \leqslant i<j \leqslant n$ such that $\left[x_{i}, x_{j}\right] \neq 1$. Since $k_{2}$ is odd, $k_{2}-1$ is even, which together with the fact $k_{2}$ divides $r_{i}$ for all $i \in\{1,2, \ldots, n\}$, implies that $k_{2}$ divides $k_{1} s_{i, j}$. Since $k_{1}$ and $k_{2}$ are coprime, this implies that $k_{2}$ divides $s_{i, j}$. Therefore

$$
u_{i, j}=\frac{s_{i, j}}{k_{2}}+\frac{1}{2}\left(k_{2}-1\right) t_{i} t_{j}
$$

is an integer for all $1 \leqslant i<j \leqslant n$ such that $\left[x_{i}, x_{j}\right] \neq 1$.

So $k_{2}$ odd implies that $g=\left(g^{\prime}\right)^{k_{2}}$ for some $g^{\prime} \in G$, which in turn implies that $h=\left(g^{\prime}\right)^{k_{1}}$ since $g^{k_{1}}=h^{k_{2}}$.

Similarly, if $k_{1}$ is odd then $h=\left(h^{\prime}\right)^{k_{1}}$ for some $h^{\prime} \in G$, so that $g=\left(h^{\prime}\right)^{k_{2}}$. Since $\left(k_{1}, k_{2}\right)=1$ implies that at least one of $k_{1}, k_{2}$ is odd, if $k_{1}$ and $k_{2}$ are coprime, $g, h$ must both be powers of a common element.

We have thus established that for any $k_{1}, k_{2} \in \mathbb{N}$, if $g^{k_{1}}=h^{k_{2}}$ then $g$ and $h$ are both powers of a common element.

We say $h$ is an (nth) root of $g$ if there exists a positive integer $n$ such that $h^{n}=g$. We call $h$ a least root of $g$ if $g=h^{n}$ for some $n \in \mathbb{N}$, and for every $w \in G$ such that $w^{m}=g$ for some $m \in \mathbb{N}$ we have $m \mid n$ and $w=h^{\frac{n}{m}}$.

LEMMA. Every element $g \in G$ has a unique least root.

Proof. Let

$$
g=x_{1}^{r_{1}} \ldots x_{n}^{r_{n}} \prod_{\substack{1 \leqslant i<j \leqslant n \\\left[x_{i}, x_{j}\right] \neq 1}}\left[x_{i}, x_{j}\right]^{s_{i, j}}, \quad h=x_{1}^{r_{1}^{\prime}} \ldots x_{n}^{r_{n}^{\prime}} \prod_{\substack{1 \leqslant i<j \leqslant n \\\left[x_{i}, x_{j}\right] \neq 1}}\left[x_{i}, x_{j}\right]^{s_{i, j}^{\prime}},
$$

be normal forms for elements $g, h \in G$. Notice that if $g$ has a least root, it must be unique, since we have already shown that if $u^{k}=v^{k}$ for elements $u, v$ of $G$ then $u=v$. We now show that every element $g$ of $G$ has a least root.

First, we note that $h^{m}=g$ implies that:

(i) $r_{i}=m r_{i}^{\prime}$ for all $i \in\{1,2, \ldots, n\}$; and,

(ii) for all $1 \leqslant i<j \leqslant n$ such that $\left[x_{i}, x_{j}\right] \neq 1$, we have

$$
s_{i, j}=m s_{i, j}^{\prime}-\frac{1}{2}(m-1) m r_{i}^{\prime} r_{j}^{\prime}
$$


Let the greatest common divisor of $r_{1}, r_{2}, \ldots, r_{n}$ be $k$. If $k=1$, then $g$ is its own least root. If $k$ is greater than one, let $d$ be the greatest divisor of $k$ such that there exist integers $u_{i, j}$ satisfying

$$
s_{i, j}=d u_{i, j}-\frac{d-1}{2 d} r_{i} r_{j}
$$

for all $i, j \in\{1,2, \ldots, n\}$ such that $\left[x_{i}, x_{j}\right] \neq 1$. In other words, let $d$ be the greatest divisor of $k$ such that

$$
\frac{s_{i, j}}{d}+\frac{d-1}{2 d^{2}} r_{i} r_{j}
$$

is an integer for all $i, j \in\{1,2, \ldots, n\}$ such that $\left[x_{i}, x_{j}\right] \neq 1$. If $d=1$, then $g$ is its own least root. For $d>1, g$ has a $d$ th root

$$
h=x_{1}^{\frac{r_{1}}{d}} x_{2}^{\frac{r_{2}}{d}} \ldots x_{n}^{\frac{r_{n}}{d}} \prod_{\substack{1 \leqslant i<j \leqslant n \\\left[x_{i}, x_{j}\right] \neq 1}}\left[x_{i}, x_{j}\right]^{\frac{s_{i j}}{d}+\frac{d-1}{2 d^{2}} r_{i} r_{j}},
$$

and $h$ is its own least root by definition of $d$. Now, for any root $w$ of $g$, we have $g=w^{r}=h^{d}$ for some $r \in \mathbb{Z}$, so that $w$ and $h$ are both powers of some common element. Since $h$ is its own least root, this means that $h$ must be a root of $w$, say $w=h^{s}$. Now $w^{r}=h^{s r}=h^{d}$ implies that $d=r s$, so $r \mid d$ and $w=h^{\frac{d}{r}}$. Therefore $h$ is a least root of $g$.

4. Centralisers. Let $S$ be a subset of a group $G$. The centraliser of $S$ in $G$ is denoted by

$$
C_{G}(S)=\{x \in G \mid s x=x s \quad \forall s \in S\} .
$$

The centraliser in $G$ of an element $g \in G$ is

$$
C_{G}(g)=\{h \in G \mid g h=h g\} .
$$

The centre of $G$ is denoted by $Z(G)$.

Let $G$ be a partially commutative nilpotent group of class 2,

$$
G=\left\langle x_{1}, x_{2}, \ldots, x_{n} \mid\left\{\left[\left[x_{i}, x_{j}\right], x_{k}\right]: i, j, k \in\{1,2, \ldots, n\}\right\},\left[x_{i_{1}}, x_{j_{1}}\right], \ldots,\left[x_{i_{m}}, x_{j_{m}}\right]\right\rangle,
$$

and let $g, h$ be elements of $G$ :

$$
g=x_{1}^{r_{1}} x_{2}^{r_{2}} \ldots x_{n}^{r_{n}} \prod_{\substack{1 \leqslant i<j \leqslant n \\\left[x_{i}, x_{j}\right] \neq 1}}\left[x_{i}, x_{j}\right]^{s_{i j}}, \quad h=x_{1}^{r_{1}^{\prime}} x_{2}^{r_{2}^{\prime}} \ldots x_{n}^{r_{n}^{\prime}} \prod_{\substack{1 \leqslant i<j \leqslant n \\\left[x_{i}, x_{j} \neq \neq 1\right.}}\left[x_{i}, x_{j}\right]^{s_{i j}} .
$$

Note that

$$
g h=x_{1}^{r_{1}+r_{1}^{\prime}} x_{2}^{r_{2}+r_{2}^{\prime}} \ldots x_{n}^{r_{n}+r_{n}^{\prime}} \prod_{\substack{1 \leqslant i<j \leqslant n \\\left[x_{i}, x_{j}\right] \neq 1}}\left[x_{i}, x_{j}\right]^{s_{i j}+s_{i, j}^{\prime}-r_{i}^{\prime} r_{j}}
$$


and

$$
h g=x_{1}^{r_{1}^{\prime}+r_{1}} x_{2}^{r_{2}^{\prime}+r_{2}} \ldots x_{n}^{r_{n}^{\prime}+r_{n}} \prod_{\substack{1 \leqslant i<j \leqslant n \\\left[x_{i}, x_{j}\right] \neq 1}}\left[x_{i}, x_{j}\right]^{s_{i, j}^{\prime}+s_{i, j}-r_{i} r_{j}^{\prime}}
$$

Therefore $g$ commutes with $h$ precisely when $r_{i}^{\prime} r_{j}=r_{i} r_{j}^{\prime}$ for all $i, j$ such that $1 \leqslant i<j \leqslant n$ and $\left[x_{i}, x_{j}\right] \neq 1$, and the centraliser of $g$ in $G$ is

$$
C_{G}(g)=\left\{h \in G \mid r_{i}^{\prime} r_{j}=r_{i} r_{j}^{\prime} \text { for } 1 \leqslant i<j \leqslant n \text { with }\left[x_{i}, x_{j}\right] \neq 1\right\} .
$$

We may note that $r_{i}^{\prime} r_{j}=r_{i} r_{j}^{\prime}$ if and only if:

(i) $r_{i}=0, r_{j} \neq 0 \Rightarrow r_{i}^{\prime}=0$;

(ii) $r_{i} \neq 0, r_{j}=0 \Rightarrow r_{j}^{\prime}=0$; and,

(iii) $r_{i} \neq 0, r_{j} \neq 0 \Rightarrow \frac{r_{i}^{\prime}}{r_{i}}=\frac{r_{j}^{\prime}}{r_{j}}$.

In the case where $g$ is a generator, say $g=x_{k}$ for some $k \in\{1,2, \ldots, n\}$, we have

$$
C_{G}\left(x_{k}\right)=\left\{h \in G \mid r_{i}^{\prime}=0 \text { for } 1 \leqslant i \leqslant n \text { with }\left[x_{i}, x_{k}\right] \neq 1\right\} .
$$

Returning to the general case, we define the set $A_{g}=\left\{x_{i} \mid r_{i} \neq 0\right\}$, and split $A_{g}$ into distinct subsets $A_{g, 1}, A_{g, 2}, \ldots, A_{g, q}$, such that two distinct elements $x_{i}, x_{j} \in A_{g}$ are in the same subset if and only if either:

(i) $\left[x_{i}, x_{j}\right] \neq 1$; or,

(ii) there exist $x_{\alpha_{1}}, x_{\alpha_{2}}, \ldots, x_{\alpha_{t}} \in A_{g}$ such that $\left[x_{i}, x_{\alpha_{1}}\right] \neq 1,\left[x_{\alpha_{t}}, x_{j}\right] \neq 1$, and $\left[x_{\alpha_{k}}, x_{\alpha_{k+1}}\right] \neq 1$ for all $k \in\{1,2, \ldots, t-1\}$.

Recall that the non-commutation graph of a partially commutative nilpotent group $H=\langle X \mid R\rangle$ is the graph with vertex set $X$, and with an edge connecting vertices $x_{i}$ and $x_{j}$ if and only if $\left[x_{i}, x_{j}\right] \notin R$. Therefore the $A_{g, i}$ correspond to the vertex sets of the connected components of non-commutation graph $\Gamma\left(\left\langle A_{g}\right\rangle\right)$.

Note that $h$ is an element of the centraliser $C_{G}(g)$ if and only if:

(i) if $x_{i} \notin A_{g}$ and $x_{j} \in A_{g}$ with $\left[x_{i}, x_{j}\right] \neq 1$, then $r_{i}^{\prime}=0$; and

(ii) if $A_{g, \gamma}=\left\{x_{i_{1}}, x_{i_{2}}, \ldots, x_{i_{p}}\right\}$, then

$$
\frac{r_{i_{1}}^{\prime}}{r_{i_{1}}}=\frac{r_{i_{2}}^{\prime}}{r_{i_{2}}}=\cdots=\frac{r_{i_{p}}^{\prime}}{r_{i_{p}}}
$$

and therefore there exists $k \in \mathbb{Q}$ such that $r_{i_{j}}^{\prime}=k r_{i_{j}}$ for all $j \in\{1,2, \ldots, p\}$.

We may now write $C_{G}(g)$ in the following fashion:

$$
\begin{aligned}
C_{G}(g)= & \left\{x_{1}^{k_{1} r_{1}+m_{1}} x_{2}^{k_{2} r_{2}+m_{2}} \ldots x_{n}^{k_{n} r_{n}+m_{n}} \prod\left[x_{i}, x_{j}\right]^{s_{i, j}^{\prime}} \mid\right. \\
& \left(\left(k_{i} r_{i}\right), m_{i}, s_{i, j}^{\prime} \in \mathbb{Z} \forall i, j\right),\left(x_{i}, x_{j} \in A_{g, \gamma} \Rightarrow k_{i}=k_{j}\right), \\
& \left.\left(m_{i} \neq 0 \Rightarrow\left(x_{i} \notin A_{g},\left[x_{i}, x_{j}\right]=1 \forall x_{j} \in A_{g}\right)\right)\right\} .
\end{aligned}
$$

In the case where $G$ is a free nilpotent group of class 2 , this simplifies to

$$
\begin{aligned}
C_{G}(g)= & \left\{x_{1}^{k r_{1}+m_{1}} x_{2}^{k r_{2}+m_{2}} \ldots x_{n}^{k r_{n}+m_{n}} \prod\left[x_{i}, x_{j}\right]^{s_{i, j}^{\prime}} \mid\right. \\
& \left.\left(k r_{i}, m_{i}, s_{i, j}^{\prime} \in \mathbb{Z} \forall i, j\right),\left(A_{g} \neq \emptyset \Rightarrow m_{j}=0 \forall j\right)\right\} .
\end{aligned}
$$


Returning to the general case, notice that, by definition, the distinct $A_{g, i}$ commute pairwise. This allows us to rewrite $g$ in the form,

$$
g=u_{1} u_{2} \ldots u_{q} \prod_{\substack{1 \leqslant i<j \leqslant n \\\left[x_{i}, x_{j}\right] \neq 1}}\left[x_{i}, x_{j}\right]^{s_{i, j}},
$$

where $u_{i}=x_{1}^{t_{i, 1}} x_{2}^{t_{i, 2}} \ldots x_{n}^{t_{i, n}}$ with

$$
t_{i, j}= \begin{cases}r_{j} & \text { if } x_{j} \in A_{g, i}, \\ 0 & \text { otherwise }\end{cases}
$$

Let $p \in\{1,2, \ldots, q\}$, and let $d$ be the greatest common divisor of $t_{p, 1}, t_{p, 2}, \ldots, t_{p, n}$. For each $j \in\{1,2, \ldots, n\}$, let $c_{p, j} \in \mathbb{Z}$ be such that $t_{p, j}=d c_{p, j}$. Then,

$$
\begin{aligned}
u_{p} & =x_{1}^{d c_{p, 1}} x_{2}^{d c_{p, 2}} \ldots x_{n}^{d c_{p, n}}, \\
& =\left(x_{1}^{c_{p, 1}} x_{2}^{c_{p, 2}} \ldots x_{n}^{c_{p, n}}\right)^{d} \prod_{1 \leqslant i<j \leqslant n}\left[x_{i}, x_{j}\right]^{\frac{1}{2}(d-1) d c_{i} c_{j}} .
\end{aligned}
$$

Let $v_{p}=x_{1}^{c_{p, 1}} x_{2}^{c_{p, 2}} \ldots x_{n}^{c_{p, n}}$, and note that $C_{G}\left(u_{p}\right)=C_{G}\left(v_{p}\right)$, so that, in particular $\left[v_{i}, v_{j}\right]=$ 1 for all $i, j$. Further note that we may rewrite the centraliser $C_{G}(g)$ in the following fashion

$$
\begin{aligned}
C_{G}(g)= & \left\{v_{1}^{k_{1}} v_{2}^{k_{2}} \ldots v_{q}^{k_{q}} x_{1}^{m_{1}} x_{2}^{m_{2}} \ldots x_{n}^{m_{n}} \prod\left[x_{i}, x_{j}\right]^{s_{i, j}} \mid k_{i}, m_{i}, s_{i, j}^{\prime} \in \mathbb{Z},\right. \\
& \left.m_{i} \neq 0 \Rightarrow\left(x_{i} \notin A_{g},\left[x_{i}, x_{j}\right]=1 \forall x_{j} \in A_{g}\right)\right\} .
\end{aligned}
$$

From this, we can see that $C_{G}(g) \subseteq C_{G}\left(v_{i}\right)=C_{G}\left(u_{i}\right)$ for all $i \in\{1,2, \ldots, q\}$, so that

$$
C_{G}(g) \subseteq C_{G}\left(u_{1}\right) \cap C_{G}\left(u_{2}\right) \cap \cdots \cap C_{G}\left(u_{q}\right) .
$$

Conversely, if $h$ commutes with $u_{i}$ for all $i \in\{1,2, \ldots, q\}$, then $h$ commutes with $g=$ $u_{1} u_{2} \ldots u_{q} \prod\left[x_{i}, x_{j}\right]^{s_{i, j}}$. Therefore

$$
\begin{aligned}
C_{G}(g) & =C_{G}\left(u_{1}\right) \cap C_{G}\left(u_{2}\right) \cap \cdots \cap C_{G}\left(u_{q}\right), \\
& =C_{G}\left(v_{1}\right) \cap C_{G}\left(v_{2}\right) \cap \cdots \cap C_{G}\left(v_{q}\right) .
\end{aligned}
$$

Note in particular that this implies $C_{G}\left(g^{m}\right)=C_{G}(g)$ for all non-zero integers $m$.

We now wish to consider centralisers of subsets of $G$. For each $\alpha \in\{1,2, \ldots, p\}$, let

$$
g_{\alpha}=x_{1}^{r_{\alpha, 1}} x_{2}^{r_{\alpha, 2}} \ldots x_{n}^{r_{\alpha, n}} \prod_{\substack{1 \leqslant i<j \leqslant n \\\left[x_{i}, x_{j}\right] \neq 1}}\left[x_{i}, x_{j}\right]^{s_{\alpha, i, j}} .
$$

Then

$$
\begin{aligned}
C_{G}\left(g_{1}, g_{2}, \ldots, g_{p}\right)= & C_{G}\left(g_{1}\right) \cap C_{G}\left(g_{2}\right) \cap \cdots \cap C_{G}\left(g_{p}\right) \\
= & \left\{x_{1}^{r_{1}} x_{2}^{r_{2}} \ldots x_{n}^{r_{n}} \prod\left[x_{i}, x_{j}\right]^{s_{i, j}} \mid r_{\alpha, i} r_{j}=r_{i} r_{\alpha, j} \text { for } 1 \leqslant \alpha \leqslant p,\right. \\
& \left.1 \leqslant i<j \leqslant n \text { with }\left[x_{i}, x_{j}\right] \neq 1\right\} .
\end{aligned}
$$

Let $A=A_{g_{1}} \cup A_{g_{2}} \cup \cdots \cup A_{g_{p}}$. 
Then

$$
\begin{aligned}
C_{G}\left(g_{1}, g_{2}, \ldots, g_{p}\right)= & \left\{x_{1}^{r_{1}} x_{2}^{r_{2}} \ldots x_{n}^{r_{n}} \prod\left[x_{i}, x_{j}\right]^{s_{i, j}} \mid\right. \\
& \left(x_{i}, x_{j} \in A_{g_{\alpha}, \gamma} \Rightarrow r_{i}=k_{\alpha, \gamma} r_{\alpha, i}, r_{j}=k_{\alpha, \gamma} r_{\alpha, j}\right), \\
& \left.\left(x_{i} \notin A, x_{j} \in A \text { with }\left[x_{i}, x_{j}\right] \neq 1 \Rightarrow r_{i}=0\right)\right\} .
\end{aligned}
$$

5. Centraliser Dimension. Let $G$ be a group. The centraliser dimension, $\operatorname{cdim}(G)$, of $G$ is the maximum length of a strict chain of centralisers of subsets of $G$. Where no finite maximum exists, $G$ is said to have infinite centraliser dimension. Thus if $G$ had a strict centraliser chain of length $n$,

$$
G=C_{G}(1) \supsetneq C_{G}\left(S_{1}\right) \supsetneq C_{G}\left(S_{2}\right) \supsetneq \cdots \supsetneq C_{G}\left(S_{n}\right)=Z(G),
$$

then $\operatorname{cdim}(G) \geqslant n$. If $\operatorname{cdim}(G)=n$ then there exist elements $g_{1}, g_{2}, \ldots, g_{n} \in G$ such that

$$
G=C_{G}(1) \supsetneq C_{G}\left(g_{1}\right) \supsetneq C_{G}\left(g_{1}, g_{2}\right) \supsetneq \cdots \supsetneq C_{G}\left(g_{1}, g_{2}, \ldots, g_{n}\right)=Z(G) .
$$

Suppose $G$ is free nilpotent of class 2 ;

$$
G=\left\langle x_{1}, x_{2}, \ldots, x_{n} \mid\left\{\left[\left[x_{i}, x_{j}\right], x_{k}\right]: i, j, k \in\{1,2, \ldots, n\}\right\}\right\rangle .
$$

Then the centre of $G$ is

$$
Z(G)=\left\{\prod_{1 \leqslant i<j \leqslant n}\left[x_{i}, x_{j}\right]^{s_{i, j}} \mid s_{i, j} \in \mathbb{Z}\right\} .
$$

Let $g_{1}, g_{2}$ be elements of $G$, where each $g_{\alpha}$ has normal form

$$
g_{\alpha}=x_{1}^{r_{\alpha, 1}} x_{2}^{r_{\alpha, 2}} \ldots x_{n}^{r_{\alpha, n}} \prod_{1 \leqslant i<j \leqslant n}\left[x_{i}, x_{j}\right]^{s_{\alpha, i, j}} .
$$

The centraliser of $g_{1}$ in $G$ is

$$
\begin{aligned}
C_{G}\left(g_{1}\right)= & \left\{x_{1}^{k r_{1,1}+m_{1}} x_{2}^{k r_{1,2}+m_{2}} \ldots x_{n}^{k r_{1, n}+m_{n}} \prod\left[x_{i}, x_{j}\right]^{s_{i, j}} \mid s_{i, j}, k r_{1, i}, m_{i} \in \mathbb{Z},\right. \\
& \left.\left(r_{1, i} \neq 0 \Rightarrow m_{j}=0 \forall j\right)\right\}, \\
= & \begin{cases}\left\{x_{1}^{k r_{1,1}} x_{2}^{k r_{1,2}} \ldots x_{n}^{k r_{1, n}} \prod\left[x_{i}, x_{j}\right]^{s_{i, j}} \mid s_{i, j},\left(k r_{1, i}\right) \in \mathbb{Z}\right\} & \text { for } g_{1} \notin Z(G), \\
G & \text { for } g_{1} \in Z(G),\end{cases}
\end{aligned}
$$

and if $C_{G}\left(g_{1}\right) \neq G$ then

$$
C_{G}\left(g_{1}, g_{2}\right)= \begin{cases}C_{G}\left(g_{1}\right) & \text { if } \exists k \in \mathbb{Q} \text { such that } k r_{1, i}=r_{2, i} \forall i, \\ Z(G) & \text { otherwise. }\end{cases}
$$

Thus the length of a centraliser chain can be no greater than two. For any $g \in G$ which is not an element of the centre of $G$ we have a maximal chain

$$
G=C_{G}(1) \supsetneq C_{G}(g) \supsetneq Z(G)
$$


and the centraliser dimension of a free nilpotent group, $G$, of class 2 is two.

In [5] it is shown that the centraliser dimension of a partially commutative group $G=\langle X \mid R\rangle$ is equal to the maximum possible length of a strictly descending chain of centralisers of subsets of $X$. We will now show that this is also the case for partially commutative nilpotent groups of class 2 .

Proposition. Let $G=\langle X \mid R\rangle$ be a partially commutative nilpotent group of class 2 . Then the centraliser dimension of $G$ is finite. Further, if $\operatorname{cdim}(G)=d$ then there exist elements $x_{i_{1}}, x_{i_{2}}, \ldots, x_{i_{d}}$ of $X$ such that

$$
G \supsetneq C_{G}\left(x_{i_{1}}\right) \supsetneq C_{G}\left(x_{i_{1}}, x_{i_{2}}\right) \supsetneq \cdots \supsetneq C_{G}\left(x_{i_{1}}, x_{i_{2}}, \ldots, x_{i_{d}}\right)=Z(G)
$$

is a centraliser chain in $G$.

Proof. Let

$$
G=C_{G}(1) \supsetneq C_{G}\left(g_{1}\right) \supsetneq C_{G}\left(g_{1}, g_{2}\right) \supsetneq \ldots \supsetneq C_{G}\left(g_{1}, g_{2}, \ldots, g_{d}\right)
$$

be a centraliser chain in $G$. We wish to show that this implies there exist elements $x_{i_{1}}, x_{i_{2}}, \ldots, x_{i_{d}}$ in $X$ such that

$$
G \supsetneq C_{G}\left(x_{i_{1}}\right) \supsetneq C_{G}\left(x_{i_{1}}, x_{i_{2}}\right) \supsetneq \cdots \supsetneq C_{G}\left(x_{i_{1}}, x_{i_{2}}, \ldots, x_{i_{d}}\right)
$$

is a centraliser chain in $G$. Let $X=\left\{x_{1}, x_{2}, \ldots, x_{n}\right\}$, and for each $\alpha \in\{1,2, \ldots, d\}$, let $g_{\alpha}$ have normal form

$$
g_{\alpha}=x_{1}^{r_{\alpha, 1}} x_{2}^{r_{\alpha, 2}} \ldots x_{n}^{r_{\alpha, n}} \prod_{1 \leqslant i<j \leqslant n}\left[x_{i}, x_{j}\right]^{s_{\alpha, i, j}} .
$$

We now rewrite each $g_{\alpha}$ in the form

$$
g_{\alpha}=u_{\alpha, 1} u_{\alpha, 2} \ldots u_{\alpha, q_{\alpha}} \prod_{1 \leqslant i<j \leqslant n}\left[x_{i}, x_{j}\right]^{s_{\alpha, i, j}},
$$

where $u_{\alpha, i}=x_{1}^{t_{\alpha, i, 1}} x_{2}^{t_{\alpha, i, 2}} \ldots x_{n}^{t_{\alpha, i, n}}$ with

$$
t_{\alpha, i, j}= \begin{cases}r_{\alpha, j} & \text { if } x_{j} \in A_{g_{\alpha}, i} \\ 0 & \text { otherwise }\end{cases}
$$

Further, for each $i \in\left\{1,2, \ldots, q_{\alpha}\right\}$, we let $c_{\alpha, i}$ be the greatest common divisor of $t_{\alpha, i, 1}, t_{\alpha, i, 2}, \ldots, t_{\alpha, i, n}$, and let

$$
v_{\alpha, i}=x_{1}^{t_{\alpha, i, 1}^{\prime}} x_{2}^{t_{\alpha, i, 2}^{\prime}} \ldots x_{n}^{t_{\alpha, i, n}^{\prime}}
$$

where $t_{\alpha, i, j}=t_{\alpha, i, j}^{\prime} c_{\alpha, i}$ for all $j \in\{1,2, \ldots, n\}$. Recall that for elements written in this form,

$$
C_{G}\left(g_{\alpha}\right)=C_{G}\left(v_{\alpha, 1}\right) \cap C_{G}\left(v_{\alpha, 2}\right) \cap \cdots \cap C_{G}\left(v_{\alpha, q_{\alpha}}\right) .
$$

By (4),

$$
C_{G}\left(g_{1}, g_{2}, \ldots, g_{\alpha-1}\right) \neq C_{G}\left(g_{1}, g_{2}, \ldots, g_{\alpha}\right)=C_{G}\left(g_{1}, g_{2}, \ldots, g_{\alpha-1}\right) \cap C_{G}\left(g_{\alpha}\right),
$$


which implies that

$$
C_{G}\left(g_{1}, g_{2}, \ldots, g_{\alpha-1}\right) \nsubseteq C_{G}\left(g_{\alpha}\right) .
$$

Therefore there exists some $k \in\left\{1,2, \ldots, q_{\alpha}\right\}$ such that

$$
C_{G}\left(g_{1}, g_{2}, \ldots, g_{\alpha-1}\right) \nsubseteq C_{G}\left(v_{\alpha, k}\right) \text {. }
$$

Reorder the $v_{\alpha, i}$ as necessary so that $k=1$. Noting that $C_{G}\left(g_{\alpha}\right) \subseteq C_{G}\left(v_{\alpha, 1}\right)$, we may deduce that

$$
C_{G}\left(g_{1}, \ldots, g_{\alpha-1}\right) \supsetneq C_{G}\left(g_{1}, \ldots, g_{\alpha-1}, v_{\alpha, 1}\right) \supseteq C_{G}\left(g_{1}, \ldots, g_{\alpha-1}, g_{\alpha}\right) .
$$

Further note that,

$$
C_{G}\left(g_{1}, g_{2}, \ldots, g_{i}\right)=C_{G}\left(g_{1}, \ldots, g_{\alpha-1}, v_{\alpha, 1}, g_{\alpha+1}, \ldots, g_{i}\right) \cap C_{G}\left(v_{\alpha, 2}, v_{\alpha, 3}, \ldots, v_{\alpha, q_{\alpha}}\right),
$$

for all $i \in\{\alpha, \alpha+1, \ldots, d\}$, and therefore the chain (4) implies that

$$
\begin{aligned}
C_{G}\left(g_{1}, g_{2}, \ldots, g_{\alpha-1}, v_{\alpha, 1}\right) & \supsetneq C_{G}\left(g_{1}, g_{2}, \ldots, g_{\alpha-1}, v_{\alpha, 1}, g_{\alpha+1}, g_{\alpha+2}, \ldots, g_{j}\right) \\
& \supsetneq C_{G}\left(g_{1}, g_{2}, \ldots, g_{\alpha-1}, v_{\alpha, 1}, g_{\alpha+1}, g_{\alpha+2}, \ldots, g_{j+1}\right),
\end{aligned}
$$

for all $j \in\{\alpha+1, \alpha+2, \ldots, d-1\}$. This means that we can replace $g_{\alpha}$ by $v_{\alpha, 1}$ in the centraliser chain (4) and still have a strictly descending chain of length $d$. For each $\alpha \in$ $\{1,2, \ldots, d\}$ we therefore assume that $q_{\alpha}=1$, that $s_{\alpha, i, j}=0$ for all $i, j \in\{1,2, \ldots, n\}$, and that $r_{\alpha, 1}, r_{\alpha, 2}, \ldots, r_{\alpha, n}$ are coprime.

Now suppose that $g_{\alpha} \in C_{G}\left(g_{1}, g_{2}, \ldots, g_{d}\right)$ for some $\alpha \in\{1,2, \ldots, d\}$. For each $i \in\{1,2, \ldots, d\}$, note that $g_{i} \notin Z(G)$, and therefore there exists some $x \in X$ such that $\left[g_{i}, x\right] \neq 1$. This implies that, for some $d^{\prime}>d$, there exist elements $g_{d+1}, g_{d+2}, \ldots, g_{d^{\prime}}$ of $X$ such that

$$
G \supsetneq C_{G}\left(g_{1}\right) \supsetneq C_{G}\left(g_{1}, g_{2}\right) \supsetneq \ldots \supsetneq C_{G}\left(g_{1}, g_{2}, \ldots, g_{d^{\prime}}\right)=C,
$$

and $g_{i} \notin C$ for all $i \in\left\{1,2, \ldots, d^{\prime}\right\}$. Note that if we can replace $g_{i}$ with an element of $X$ for each $i \in\left\{1,2, \ldots, d^{\prime}\right\}$ so that this chain still holds, then it is certainly true for the shorter chain (4). Therefore we may assume that there exists no $\alpha \in\{1,2, \ldots, d\}$ such that $g_{\alpha} \in C_{G}\left(g_{1}, g_{2}, \ldots, g_{d}\right)$.

We now have a centraliser chain

$$
G=C_{G}(1) \supsetneq C_{G}\left(g_{1}\right) \supsetneq C_{G}\left(g_{1}, g_{2}\right) \supsetneq \ldots \supsetneq C_{G}\left(g_{1}, g_{2}, \ldots, g_{d}\right),
$$

where, for all $\alpha \in\{1,2, \ldots, d\}$,

(1) $g_{\alpha}=x_{1}^{r_{\alpha, 1}} x_{2}^{r_{\alpha, 2}} \ldots x_{n}^{r_{\alpha, n}}$, with $r_{\alpha, i}$ and $r_{\alpha, j}$ both non-zero only if either

(i) $\left[x_{i}, x_{j}\right] \neq 1$; or,

(ii) for some $m \neq 0$, there exist $k_{1}, k_{2}, \ldots, k_{m} \in\{1,2, \ldots, n\}$ such that

(a) $r_{\alpha, k_{l}}$ is non-zero for all $l \in\{1,2, \ldots, m\}$; and,

(b) $\left[x_{i}, x_{k_{1}}\right] \neq 1, \quad\left[x_{k_{l}}, x_{k_{l+1}}\right] \neq 1 \quad$ for $\quad$ all $l \in\{1,2, \ldots, m-1\}, \quad$ and $\left[x_{k_{m}}, x_{j}\right] \neq 1$

(2) $r_{\alpha, 1}, r_{\alpha, 2}, \ldots, r_{\alpha, n}$ are coprime, and therefore $g_{\alpha}$ is a least root; and,

(3) $g_{\alpha} \notin C_{G}\left(g_{1}, g_{2}, \ldots, g_{d}\right)$.

For each $\alpha \in\{1,2, \ldots, d\}$, define $\beta_{\alpha} \in\{1,2, \ldots, d\}$ to be minimal such that $g_{\alpha} \notin$ $C_{G}\left(g_{1}, g_{2}, \ldots, g_{\beta_{\alpha}}\right)$. 
Suppose that for some $\alpha \in\{2,3, \ldots, d\}$ we have $\beta_{\alpha}=\beta>\alpha+1$ and $g_{\alpha} \notin X^{ \pm 1}$. Since $g_{\alpha}$ is a least root, the condition that $g_{\alpha} \notin X^{ \pm 1}$ implies that there exist $i_{1}, i_{2} \in$ $\{1,2, \ldots, n\}$ such that $r_{\alpha, i_{1}}$ and $r_{\alpha, i_{2}}$ are both non-zero and $\left[x_{i_{1}}, x_{i_{2}}\right] \neq 1$. This allows us to define an element

$$
w_{\alpha}=x_{1}^{t_{\alpha, 1}} x_{2}^{t_{\alpha, 2}} \ldots x_{n}^{t_{\alpha, n}}
$$

of $G$ such that

(i) $t_{\alpha, i}=0$ if and only if $r_{\alpha, i}=0$;

(ii) $t_{\alpha, 1}, t_{\alpha, 2}, \ldots, t_{\alpha, n}$ are coprime, and therefore $w_{\alpha}$ is a least root; and,

(iii) $\left[g_{\alpha}, w_{\alpha}\right] \neq 1$.

For example, let

$$
t_{\alpha, i}^{\prime}= \begin{cases}2 r_{\alpha, i_{1}} & \text { if } i=i_{1} \\ r_{\alpha, i} & \text { otherwise }\end{cases}
$$

and let $c$ be the greatest common divisor of $t_{\alpha, 1}^{\prime}, t_{\alpha, 2}^{\prime}, \ldots, t_{\alpha, n}^{\prime}$. If we let $t_{\alpha, i}$ be such that $t_{\alpha, i}^{\prime}=c t_{\alpha, i}$ for all $i \in\{1,2, \ldots, n\}$, then $w_{\alpha}$ satisfies the required conditions. We have chosen our definition of $w_{\alpha}$ such that

(i) $A_{w_{\alpha}}=A_{g_{\alpha}}$; and,

(ii) $\left\langle g_{\alpha}\right\rangle \cap\left\langle w_{\alpha}\right\rangle=1$.

Further note that $g_{\alpha}$ is an element of $C_{G}\left(g_{1}, g_{2}, \ldots, g_{\beta-1}\right)$, but fails to commute with $w_{\alpha}$, and therefore

$$
C_{G}\left(g_{1}, g_{2}, \ldots, g_{\beta-1}\right) \supsetneq C_{G}\left(g_{1}, g_{2}, \ldots, g_{\beta-1}, w_{\alpha}\right) .
$$

Now note that

$$
\begin{gathered}
C_{G}\left(g_{\alpha}\right)=\left\langle g_{\alpha}\right\rangle \times\left\{x_{1}^{m_{1}} x_{2}^{m_{2}} \ldots x_{n}^{m_{n}} \prod\left[x_{i}, x_{j}\right]^{s_{i, j}} \mid m_{i}, s_{i, j} \in \mathbb{Z},\right. \\
\left.m_{i} \neq 0 \Rightarrow\left(\left[x_{i}, x_{j}\right]=1 \forall x_{j} \in A_{g_{\alpha}}\right)\right\},
\end{gathered}
$$

and, since $A_{g_{\alpha}}=A_{w_{\alpha}}$,

$$
\begin{gathered}
C_{G}\left(w_{\alpha}\right)=\left\langle w_{\alpha}\right\rangle \times\left\{x_{1}^{m_{1}} x_{2}^{m_{2}} \ldots x_{n}^{m_{n}} \prod\left[x_{i}, x_{j}\right]^{s_{i, j}} \mid m_{i}, s_{i, j} \in \mathbb{Z},\right. \\
\left.m_{i} \neq 0 \Rightarrow\left(\left[x_{i}, x_{j}\right]=1 \forall x_{j} \in A_{g_{\alpha}}\right)\right\} .
\end{gathered}
$$

Further $\left\langle g_{\alpha}\right\rangle \cap\left\langle w_{\alpha}\right\rangle=1$ implies that

$$
\begin{aligned}
C_{G}\left(g_{\alpha}\right) \cap C_{G}\left(w_{\alpha}\right)= & \left\{x_{1}^{m_{1}} x_{2}^{m_{2}} \ldots x_{n}^{m_{n}} \prod\left[x_{i}, x_{j}\right]^{s_{i, j}} \mid m_{i}, s_{i, j} \in \mathbb{Z},\right. \\
& \left.m_{i} \neq 0 \Rightarrow\left(\left[x_{i}, x_{j}\right]=1 \forall x_{j} \in A_{g_{\alpha}}\right)\right\}, \\
= & C_{G}\left(g_{\alpha}\right) \cap\left\{x_{1}^{r_{1}} x_{2}^{r_{2}} \ldots x_{n}^{r_{n}} \prod\left[x_{i}, x_{j}\right]^{s_{i, j}} \mid r_{\alpha, i} \neq 0 \Rightarrow r_{i}=0\right\} .
\end{aligned}
$$

Note, too, that since $g_{\alpha}$ and $g_{\beta}$ do not commute, we must have

$$
C_{G}\left(g_{\alpha}\right) \cap C_{G}\left(g_{\beta}\right) \subseteq\left\{x_{1}^{r_{1}} x_{2}^{r_{2}} \ldots x_{n}^{r_{n}} \prod\left[x_{i}, x_{j}\right]^{s_{i, j}} \mid r_{\alpha, i} \neq 0 \Rightarrow r_{i}=0\right\} .
$$


We now combine these results to see that

$$
\begin{aligned}
& C_{G}\left(g_{1}, g_{2}, \ldots, g_{\beta-1}, w_{\alpha}\right) \\
& \quad=C_{G}\left(g_{1}, g_{2}, \ldots, g_{\alpha}, \ldots, g_{\beta-1}\right) \cap C_{G}\left(w_{\alpha}\right) \\
& \quad=C_{G}\left(g_{1}, g_{2}, \ldots, g_{\beta-1}\right) \cap\left(C_{G}\left(g_{\alpha}\right) \cap C_{G}\left(w_{\alpha}\right)\right), \\
& \quad=C_{G}\left(g_{1}, \ldots, g_{\beta-1}\right) \cap\left(C_{G}\left(g_{\alpha}\right) \cap\left\{x_{1}^{r_{1}} \ldots x_{n}^{r_{n}} \prod\left[x_{i}, x_{j}\right]^{s_{i, j}} \mid r_{\alpha, i} \neq 0 \Rightarrow r_{i}=0\right\}\right), \\
& \quad=C_{G}\left(g_{1}, g_{2}, \ldots, g_{\alpha}, \ldots, g_{\beta-1}\right) \cap\left\{x_{1}^{r_{1}} \ldots x_{n}^{r_{n}} \prod\left[x_{i}, x_{j}\right]^{s_{i, j}} \mid r_{\alpha, i} \neq 0 \Rightarrow r_{i}=0\right\}, \\
& \quad \supseteq C_{G}\left(g_{1}, g_{2}, \ldots, g_{\alpha}, \ldots, g_{\beta-1}\right) \cap\left(C_{G}\left(g_{\alpha}\right) \cap C_{G}\left(g_{\beta}\right)\right), \\
& \quad=C_{G}\left(g_{1}, g_{2}, \ldots, g_{\beta}\right),
\end{aligned}
$$

and therefore,

$$
C_{G}\left(g_{1}, g_{2}, \ldots, g_{\beta-1}\right) \supsetneq C_{G}\left(g_{1}, g_{2}, \ldots, g_{\beta-1}, w_{\alpha}\right) \supseteq C_{G}\left(g_{1}, g_{2}, \ldots, g_{\beta}\right) .
$$

Note that this implies that

$$
C_{G}\left(g_{1}, g_{2}, \ldots, g_{i}\right) \subseteq C_{G}\left(g_{1}, \ldots, g_{\beta-1}, w_{\alpha}, g_{\beta+1}, \ldots, g_{i}\right)
$$

for all $i \in\{\beta+1, \beta+2, \ldots, d\}$, and further the strictness of chain (4) implies that

$$
C_{G}\left(g_{1}, g_{2}, \ldots, g_{i}\right) \nsubseteq C_{G}\left(g_{i+1}\right)
$$

for all $i \in\{1,2, \ldots, d-1\}$. This implies that

$$
C_{G}\left(g_{1}, \ldots, g_{\beta-1}, w_{\alpha}, g_{\beta+1}, \ldots, g_{i}\right) \nsubseteq C_{G}\left(g_{i+1}\right),
$$

and therefore

$$
C_{G}\left(g_{1}, \ldots, g_{\beta-1}, w_{\alpha}, g_{\beta+1}, \ldots, g_{i}\right) \supsetneq C_{G}\left(g_{1}, \ldots, g_{\beta-1}, w_{\alpha}, g_{\beta+1}, \ldots, g_{i+1}\right),
$$

for all $i \in\{\beta, \beta+1, \ldots, d-1\}$. We may therefore replace $g_{\beta}$ with $w_{\alpha}$ in the chain (4).

For $p \in\{\alpha, \alpha+1, \ldots, \beta-1\}$, note that

$$
C_{G}\left(g_{1}, g_{2}, \ldots, g_{p}\right)=C_{G}\left(g_{1}, g_{2}, \ldots, g_{p}, w_{\alpha}\right) \times\left\langle g_{\alpha}\right\rangle .
$$

Since (4) is a strict centraliser chain, so is the chain

$$
C_{G}\left(g_{1}, \ldots, g_{\alpha}, w_{\alpha}\right) \supsetneq C_{G}\left(g_{1}, \ldots, g_{\alpha+1}, w_{\alpha}\right) \supsetneq \cdots \supsetneq C_{G}\left(g_{1}, \ldots, g_{\beta-1}, w_{\alpha}\right) .
$$

Therefore

$$
\begin{aligned}
& G \supsetneq C_{G}\left(g_{1}\right) \supsetneq \cdots \supsetneq C_{G}\left(g_{1}, \ldots, g_{\alpha}\right) \supsetneq C_{G}\left(g_{1}, \ldots, g_{\alpha}, w_{\alpha}\right) \supsetneq \ldots \\
& \quad \supsetneq C_{G}\left(g_{1}, \ldots, g_{\beta-1}, w_{\alpha}\right) \supsetneq \cdots \supsetneq C_{G}\left(g_{1}, \ldots, g_{\beta-1}, g_{\beta+1}, \ldots, g_{d}, w_{\alpha}\right)
\end{aligned}
$$

is a strict centraliser chain of length $d$, and further $\beta_{\alpha}=\alpha+1$.

We apply this process to each such $\alpha$, in numerical order, to obtain a chain (4) with the property that, for all $\alpha \in\{1,2, \ldots, d\}$, either $g_{\alpha} \in X^{ \pm 1}$ or $\beta_{\alpha} \leqslant \alpha+1$. Note that if $g_{\alpha} \in X^{-1}$ then we may replace $g_{\alpha}$ with $g_{\alpha}^{-1} \in X$ since $C_{G}(g)=C_{G}\left(g^{-1}\right)$ for all $g \in G$. Therefore we may further assume that our chain (4) has the property that, for all $\alpha \in\{1,2, \ldots, d\}$, either $g_{\alpha} \in X$ or $\beta_{\alpha} \leqslant \alpha+1$. 

of $\beta_{\alpha}$ :

Suppose $g_{\alpha} \notin X$, with $g_{j} \in X$ for all $j<\alpha$. We have two possibilities for the value

(1) $\beta_{\alpha}<\alpha+1$

Note that since $C_{G}\left(g_{1}, g_{2}, \ldots, g_{\alpha-1}\right)$ and $C_{G}\left(g_{1}, g_{2}, \ldots, g_{\alpha}\right)$ are distinct, there must exist some $u \in G$ such that $\left[u, g_{i}\right]=1$ for all $i \in\{1,2, \ldots, \alpha-1\}$ and $\left[u, g_{\alpha}\right] \neq 1$. Further, $\left[u, g_{\alpha}\right] \neq 1$ implies that there exists some $k \in\{1,2, \ldots, n\}$ such that $r_{\alpha, k} \neq 0$ and $\left[u, x_{k}\right] \neq 1$. Therefore

$$
C_{G}\left(g_{1}, g_{2}, \ldots, g_{\alpha-1}\right) \supsetneq C_{G}\left(g_{1}, g_{2}, \ldots, g_{\alpha-1}, x_{k}\right) .
$$

Now

$$
\begin{aligned}
C_{G}\left(x_{k}\right) \cap & \left\{x_{1}^{r_{1}} x_{2}^{r_{2}} \ldots x_{k}^{r_{k}} \ldots x_{n}^{r_{n}} \prod\left[x_{i}, x_{j}\right]^{s_{i, j}} \mid r_{k}=0\right\} \\
& \supseteq C_{G}\left(g_{\alpha}\right) \cap\left\{x_{1}^{r_{1}} x_{2}^{r_{2}} \ldots x_{n}^{r_{n}} \prod\left[x_{i}, x_{j}\right]^{s_{i, j}} \mid r_{\alpha, i} \neq 0 \Rightarrow r_{i}=0\right\} .
\end{aligned}
$$

Therefore, since $\beta_{\alpha}<\alpha+1$ implies that $g_{\alpha}$ is not an element of the centraliser $C_{G}\left(g_{1}, g_{2}, \ldots, g_{\alpha}\right)$, we have

$$
\begin{aligned}
C_{G} & \left(g_{1}, g_{2}, \ldots, g_{\alpha-1}, x_{k}\right) \\
\supseteq & C_{G}\left(g_{1}, g_{2}, \ldots, g_{\alpha-1}\right) \cap C_{G}\left(x_{k}\right) \\
& \cap\left\{x_{1}^{r_{1}} x_{2}^{r_{2}} \ldots x_{n}^{r_{n}} \prod\left[x_{i}, x_{j}\right]^{s_{i, j}} \mid r_{k}=0\right\}, \\
\supseteq & C_{G}\left(g_{1}, g_{2}, \ldots, g_{\alpha-1}\right) \cap C_{G}\left(g_{\alpha}\right) \\
& \cap\left\{x_{1}^{r_{1}} x_{2}^{r_{2}} \ldots x_{n}^{r_{n}} \prod\left[x_{i}, x_{j}\right]^{s_{i, j}} \mid r_{\alpha, i} \neq 0 \Rightarrow r_{i}=0\right\}, \\
= & C_{G}\left(g_{1}, g_{2}, \ldots, g_{\alpha-1}, g_{\alpha}\right) .
\end{aligned}
$$

We have now shown that

$$
C_{G}\left(g_{1}, g_{2}, \ldots, g_{\alpha-1}\right) \supsetneq C_{G}\left(g_{1}, g_{2}, \ldots, g_{\alpha-1}, x_{k}\right) \supseteq C_{G}\left(g_{1}, g_{2}, \ldots, g_{\alpha}\right) .
$$

Note that if

$$
C_{G}\left(g_{1}, \ldots, g_{\alpha-1}, x_{k}, g_{\alpha+1}, \ldots, g_{i}\right)=C_{G}\left(g_{1}, \ldots, g_{\alpha-1}, x_{k}, g_{\alpha+1}, \ldots, g_{i+1}\right)
$$

for some $i \in\{\alpha, \alpha+1, \ldots, d-1\}$ then

$$
C_{G}\left(g_{1}, g_{2}, \ldots, g_{i}\right) \subseteq C_{G}\left(g_{1}, \ldots, g_{\alpha-1}, x_{k}, g_{\alpha+1}, \ldots, g_{i}\right) \subseteq C_{G}\left(g_{i+1}\right)
$$

contradicting the strictness of chain (4). We may therefore replace $g_{\alpha}$ by the generator $x_{k}$, so that (4) is a strict centraliser chain of length $d$ such that $g_{i}$ is an element of $X$ for all $i \in\{1,2, \ldots, \alpha\}$.

(2) $\beta=\beta_{\alpha}=\alpha+1$

Choose $k, l \in\{1,2, \ldots, n\}$ such that $r_{\alpha, k} \neq 0, r_{\alpha, l} \neq 0$ and $\left[x_{k}, x_{l}\right] \neq 1$. Note that $g_{\alpha} \in C_{G}\left(g_{1}, g_{2}, \ldots, g_{\alpha-1}\right)$ implies that $x_{i} \in C_{G}\left(g_{1}, g_{2}, \ldots, g_{\alpha-1}\right)$ for all $i \in$ $\{1,2, \ldots, n\}$ such that $r_{\alpha, i} \neq 0$, since $g_{j} \in X$ for all $j \in\{1,2, \ldots, \alpha-1\}$. In particular $x_{k}, x_{l} \in C_{G}\left(g_{1}, g_{2}, \ldots, g_{\alpha-1}\right)$. Therefore $x_{l} \notin C_{G}\left(x_{k}\right)$ implies that

$$
C_{G}\left(g_{1}, g_{2}, \ldots, g_{\alpha-1}\right) \supsetneq C_{G}\left(g_{1}, g_{2}, \ldots, g_{\alpha-1}, x_{k}\right),
$$


and $x_{k} \notin C_{G}\left(x_{l}\right)$ implies that

$$
C_{G}\left(g_{1}, g_{2}, \ldots, g_{\alpha-1}, x_{k}\right) \supsetneq C_{G}\left(g_{1}, g_{2}, \ldots, g_{\alpha-1}, x_{k}, x_{l}\right) .
$$

Note that

$$
\begin{aligned}
C_{G}\left(x_{k}, x_{l}\right) \cap & \left\{x_{1}^{r_{1}} x_{2}^{r_{2}} \ldots x_{n}^{r_{n}} \prod\left[x_{i}, x_{j}\right]^{s_{i, j}} \mid r_{k}=r_{l}=0\right\} \\
& \supseteq C_{G}\left(g_{\alpha}\right) \cap\left\{x_{1}^{r_{1}} x_{2}^{r_{2}} \ldots x_{n}^{r_{n}} \prod\left[x_{i}, x_{j}\right]^{s_{i, j}} \mid r_{\alpha, i} \neq 0 \Rightarrow r_{i}=0\right\},
\end{aligned}
$$

so that

$$
\begin{aligned}
C_{G} & \left(g_{1}, g_{2}, \ldots, g_{\alpha-1}, x_{k}, x_{l}\right) \\
\supseteq & C_{G}\left(g_{1}, g_{2}, \ldots, g_{\alpha-1}\right) \cap C_{G}\left(x_{k}, x_{l}\right) \\
& \cap\left\{x_{1}^{r_{1}} x_{2}^{r_{2}} \ldots x_{n}^{r_{n}} \prod\left[x_{i}, x_{j}\right]^{s_{i, j}} \mid r_{k}=r_{l}=0\right\}, \\
\supseteq & C_{G}\left(g_{1}, g_{2}, \ldots, g_{\alpha-1}\right) \cap C_{G}\left(g_{\alpha}\right) \\
\quad & \cap\left\{x_{1}^{r_{1}} x_{2}^{r_{2}} \ldots x_{n}^{r_{n}} \prod\left[x_{i}, x_{j}\right]^{s_{i, j}} \mid r_{\alpha, i} \neq 0 \Rightarrow r_{i}=0\right\} .
\end{aligned}
$$

Note further that

$$
C_{G}\left(g_{1}, g_{2}, \ldots, g_{\alpha+1}\right) \subsetneq C_{G}\left(g_{1}, g_{2}, \ldots, g_{\alpha}\right),
$$

and, since $g_{\alpha} \notin C_{G}\left(g_{1}, g_{2}, \ldots, g_{\alpha}, g_{\alpha+1}\right)$, that

$$
C_{G}\left(g_{1}, g_{2}, \ldots, g_{\alpha+1}\right) \subseteq\left\{x_{1}^{r_{1}} x_{2}^{r_{2}} \ldots x_{n}^{r_{n}} \prod\left[x_{i}, x_{j}\right]^{s_{i, j}} \mid r_{\alpha, i} \neq 0 \Rightarrow r_{i}=0\right\} .
$$

Therefore

$$
C_{G}\left(g_{1}, g_{2}, \ldots, g_{\alpha-1}, x_{k}, x_{l}\right) \supseteq C_{G}\left(g_{1}, g_{2}, \ldots, g_{\alpha+1}\right) .
$$

We have now shown that

$$
\begin{aligned}
& C_{G}\left(g_{1}, g_{2}, \ldots, g_{\alpha-1}\right) \supsetneq C_{G}\left(g_{1}, g_{2}, \ldots, g_{\alpha-1}, x_{k}\right) \\
& \quad \supsetneq C_{G}\left(g_{1}, g_{2}, \ldots, g_{\alpha-1}, x_{k}, x_{l}\right) \supseteq C_{G}\left(g_{1}, g_{2}, \ldots, g_{\alpha-1}, g_{\alpha}, g_{\alpha+1}\right) .
\end{aligned}
$$

Note, in a similar fashion to case (1), that the strictness of (4) implies that

$$
\begin{aligned}
& C_{G}\left(g_{1}, g_{2}, \ldots, g_{\alpha-1}, x_{k}, x_{l}, g_{\alpha+2}, \ldots, g_{i}\right) \\
& \quad \supsetneq C_{G}\left(g_{1}, g_{2}, \ldots, g_{\alpha-1}, x_{k}, x_{l}, g_{\alpha+2}, \ldots, g_{i+1}\right)
\end{aligned}
$$

for all $i \in\{\alpha+1, \alpha+2, \ldots, d-1\}$. We may therefore replace the existing $g_{\alpha}$ and $g_{\alpha+1}$ by $x_{k}$ and $x_{l}$ respectively to give a chain (4) for which $g_{i} \in X$ for all $i \in\{1,2, \ldots, \alpha\}$.

Repeat for each $\alpha$ such that $g_{\alpha} \notin X$.

We have now shown that for any strict centraliser chain in $G$ of finite length, $d$, there exist generators $x_{i_{1}}, x_{i_{2}}, \ldots, x_{i_{d}}$ of $X$ such that

$$
G \supsetneq C_{G}\left(x_{i_{1}}\right) \supsetneq C_{G}\left(x_{i_{1}}, x_{i_{2}}\right) \supsetneq \cdots \supsetneq C_{G}\left(x_{i_{1}}, x_{i_{2}}, \ldots, x_{i_{d}}\right) .
$$


This implies that the centraliser dimension of $G$ is bounded above by $|X|$, and is therefore finite. Further there exist $\operatorname{cdim}(G)$ generators in $X$ which give a maximal chain.

Recall that the non-commutation graph of a partially commutative or partially commutative nilpotent group $G=\langle X \mid R\rangle$ is the graph with vertex set $X$, and with an edge connecting $x_{i}$ and $x_{j}$ if and only if $\left[x_{i}, x_{j}\right] \notin R$.

THEOREM. Let $G=\left\langle x_{1}, x_{2}, \ldots, x_{n} \mid R\right\rangle$ be a partially commutative nilpotent group of class 2, with non-commutation graph $\Gamma$. Let $G^{\prime}=\left\langle x_{1}, x_{2}, \ldots, x_{n} \mid R^{\prime}\right\rangle$ be the partially commutative group with non-commutation graph $\Gamma$. Then $\operatorname{cdim}(G)=\operatorname{cdim}\left(G^{\prime}\right)$.

Proof.

(1) Let

$$
C_{G^{\prime}}\left(x_{i_{1}}, x_{i_{2}}, \ldots, x_{i_{m}}\right) \supsetneq C_{G^{\prime}}\left(x_{i_{1}}, x_{i_{2}}, \ldots, x_{i_{m+1}}\right)
$$

for some elements $x_{i_{j}} \in\left\{x_{1}, x_{2}, \ldots, x_{n}\right\}$ and some $m \geqslant 0$. This implies that there exists some $g \in G^{\prime}$ such that

(i) $\left[g, x_{i_{j}}\right]_{G^{\prime}}=1$ for all $1 \leqslant j \leqslant m$; and,

(ii) $\left[g, x_{i_{m+1}}\right]_{G^{\prime}} \neq 1$.

In [6], it is shown that if $H$ is a free partially commutative group and $x, h \in H$ with $x$ a generator, then we have $[x, h]_{H}=1$ if and only if $\left[x, x^{\prime}\right]_{H}=1$ for all generators $x^{\prime}$ which occur in $h$ when written as a product of generators of minimal length. Therefore, there exists some generator $y \in\left\{x_{1}, x_{2}, \ldots, x_{n}\right\}$ such that

(i) $\left[y, x_{i_{j}}\right]_{G^{\prime}}=1$ for all $1 \leqslant j \leqslant m$; and,

(ii) $\left[y, x_{i_{m+1}}\right]_{G^{\prime}} \neq 1$.

Since $G$ and $G^{\prime}$ have the same non-commutation graph, we also have

(i) $\left[y, x_{i_{j}}\right]_{G}=1$ for all $1 \leqslant j \leqslant m$, and,

(ii) $\left[y, x_{i_{m+1}}\right]_{G} \neq 1$.

Therefore $y \in C_{G}\left(x_{i_{1}}, x_{i_{2}}, \ldots, x_{i_{m}}\right)$ and $y \notin C_{G}\left(x_{i_{1}}, x_{i_{2}}, \ldots, x_{i_{m+1}}\right)$, so that

$$
C_{G}\left(x_{i_{1}}, x_{i_{2}}, \ldots, x_{i_{m}}\right) \supsetneq C_{G}\left(x_{i_{1}}, x_{i_{2}}, \ldots, x_{i_{m+1}}\right) .
$$

Now let $\operatorname{cdim}\left(G^{\prime}\right)=d$. This implies that there exists a maximal centraliser chain in $G^{\prime}$ of the form,

$$
G^{\prime} \supsetneq C_{G^{\prime}}\left(x_{i_{1}}\right) \supsetneq C_{G^{\prime}}\left(x_{i_{1}}, x_{i_{2}}\right) \supsetneq \ldots \supsetneq C_{G^{\prime}}\left(x_{i_{1}}, x_{i_{2}}, \ldots, x_{i_{d}}\right)
$$

for some elements $x_{i_{j}} \in\left\{x_{1}, x_{2}, \ldots, x_{n}\right\}$. Therefore

$$
G \supsetneq C_{G}\left(x_{i_{1}}\right) \supsetneq C_{G}\left(x_{i_{1}}, x_{i_{2}}\right) \supsetneq \cdots \supsetneq C_{G}\left(x_{i_{1}}, x_{i_{2}}, \ldots, x_{i_{d}}\right),
$$

is a centraliser chain in $G$, and $\operatorname{cdim}(G) \geqslant d=\operatorname{cdim}\left(G^{\prime}\right)$.

(2) Let

$$
C_{G}\left(x_{i_{1}}, x_{i_{2}}, \ldots, x_{i_{m}}\right) \supsetneq C_{G}\left(x_{i_{1}}, x_{i_{2}}, \ldots, x_{i_{m+1}}\right)
$$

for some elements $x_{i_{j}} \in\left\{x_{1}, x_{2}, \ldots, x_{n}\right\}$ and some $m \geqslant 0$. Then there exists some element $g$ of $G$ such that

(i) $\left[g, x_{i j}\right]_{G}=1$ for all $1 \leqslant j \leqslant m$; and,

(ii) $\left[g, x_{i_{m+1}}\right]_{G} \neq 1$. 
Recall that for $x \in\left\{x_{1}, x_{2}, \ldots, x_{n}\right\}$, we have

$$
C_{G}(x)=\left\{x_{1}^{r_{1}} x_{2}^{r_{2}} \ldots x_{n}^{r_{n}} \prod\left[x_{i}, x_{j}\right]_{G}^{s_{i, j}} \mid r_{i}, s_{i, j} \in \mathbb{Z}, r_{i}=0 \text { if }\left[x, x_{i}\right]_{G} \neq 1\right\} .
$$

Therefore, if $g$ has normal form $g=x_{1}^{r_{1}} x_{2}^{r_{2}} \ldots x_{n}^{r_{n}} \prod\left[x_{i}, x_{j}\right]_{G}^{s_{i j}}$ then

(i) $r_{i}=0$ for all $i \in\{1,2, \ldots, n\}$ such that $\left[x_{i}, x_{i_{k}}\right]_{G} \neq 1$ for some $k \in$ $\{1,2, \ldots, m\}$; and,

(ii) $r_{l} \neq 0$ for some $l \in\{1,2, \ldots, n\}$ such that $\left[x_{l}, x_{i_{m+1}}\right]_{G} \neq 1$.

Note that $r_{l} \neq 0$ implies $\left[x_{l}, x_{i_{j}}\right]_{G}=1$ for all $j \in\{1,2, \ldots, m\}$. Since $G$ and $G^{\prime}$ share the same non-commutation graph, we have now shown that $x_{l} \in$ $C_{G^{\prime}}\left(x_{i_{1}}, x_{i_{2}}, \ldots, x_{i_{m}}\right)$ but $x_{l} \notin C_{G^{\prime}}\left(x_{i_{1}}, x_{i_{2}}, \ldots, x_{i_{m+1}}\right)$. Therefore

$$
C_{G^{\prime}}\left(x_{i_{1}}, x_{i_{2}}, \ldots, x_{i_{m}}\right) \supsetneq C_{G^{\prime}}\left(x_{i_{1}}, x_{i_{2}}, \ldots, x_{i_{m+1}}\right) .
$$

Now suppose that $\operatorname{cdim}(G)=d$. By the same method used in part (1) of the proof, we must have $\operatorname{cdim}\left(G^{\prime}\right) \geqslant d=\operatorname{cdim}(G)$.

We thus obtain the required $\operatorname{result}, \operatorname{cdim}(G)=\operatorname{cdim}\left(G^{\prime}\right)$.

\section{REFERENCES}

1. G. Baumslag, A. Myasnikov and V. Remeslennikov, Algebraic geometry over groups I. Algebraic sets and ideal theory, J. Algebra 219 (1999), 16-79. 371-383.

2. R. M. Bryant, Groups with the minimal condition on centralizers, J. Algebra 60 (1979),

3. I. M. Chiswell and V. N. Remeslennikov, Equations in free groups with one variable. I, J. Group Theory 3(4) (2000), 445-466.

4. A. J. Duncan, I. V. Kazachkov and V. N. Remeslennikov, Centraliser dimension and universal classes of groups, Siberian Electronic Mathematical Reports 3 (2006), 197-215.

5. A. J. Duncan, I. V. Kazachkov and V. N. Remeslennikov, Centraliser dimension of partially commutative groups, Geometriae Dedicata 120 (2006), 73-97.

6. E. S. Esyp, I. V. Kazatchkov and V. N. Remeslennikov, Divisibility theory and complexity of algorithms for free partially commutative groups, Contemporary Mathematics 378 (2005), 319-348.

7. M. Hall, The theory of groups (The Macmillan Company, 1959).

8. P. Hall, Some word problems, J. London Math. Soc. 33 (1958), 482-496.

9. O. Kharlampovich and A. Myasnikov, Elementary theory of free non-abelian groups, J. Algebra 302 (2006), 451-552.

10. A. Kvaschuk, A. Myasnikov and V. Remeslennikov, Algebraic geometry over groups III. Elements of model theory, J. Algebra 288 (2005), 78-98.

11. J. C. Lennox and J. E. Roseblade, Centrality in finitely generated soluble groups, $J$. Algebra 16 (1970), 399-435.

12. A. Myasnikov and V. Remeslennikov, Algebraic geometry over groups II. Logical foundations, J. Algebra 234 (2000), 225-276.

13. A. Myasnikov and P. Schumyatsky, Discriminating groups and c-dimension, J. Group Theory 7 (2004), 135-142.

14. H. Neumann, Varieties of groups (Springer-Verlag, 1967).

15. R. Schmidt, Zentralisatorverbände endlicher gruppen, Rend. Sem. Mat. Univ. Padova 44 (1970), 97-131.

16. Z. Sela, Diophantine geometry over groups VI: the elementary theory of a free group, Geometric and Functional Analysis 16(3) (2006), 707-730. 